\title{
Active transport across the bacterial outer membrane: The Ton motor complex
}

Susan K. Buchanan $^{5 *}$, Hervé Celia ${ }^{1,5 ¥}$, Nicholas Noinaj ${ }^{2 *}$, Stanislav D. Zakharov², Enrica Bordignon $^{3,4}$, Istvan Botos 5 , Monica Santamaria ${ }^{6}$, Travis J. Barnard ${ }^{5}$, William A. Cramer², and Roland Lloubes ${ }^{1 *}$

${ }^{1}$ Laboratoire d'Ingénierie des Systèmes Macromoléculaires, UMR7255 CNRS/Aix-Marseille Université, Institut de Microbiologie de la Méditerranée, 13402 Marseille Cedex 20, France, ${ }^{2}$ Markey Center for Structural Biology, Department of Biological Sciences, and the Purdue Institute for Inflammation, Immunology and Infectious Diseases, Purdue University, West Lafayette, Indiana, 47907, ${ }^{3}$ Fachbereich Physik, Freie Universität, 14195 Berlin, Germany, ${ }^{4}$ Faculty of Chemistry and Biochemistry, Ruhr-Universität Bochum, 45810 Bochum, Germany, ${ }^{5}$ National Institute of Diabetes \& Digestive \& Kidney Diseases, Bethesda, Maryland, 20892, and ${ }^{6}$ Departamento de Cirugia Experimental, Instituto de Investigacion Hospital La Paz (IdiPAZ), Paseo de la Castellana 261, 28046 Madrid, Spain ${ }^{¥}$ Authors contributed equally.

In Gram-negative bacteria, outer membrane (OM) transporters import nutrients by coupling to an inner membrane (IM) protein complex called the Ton complex. The Ton complex consists of TonB, ExbB, and ExbD, and uses the proton motive force (pmf) at the IM to transduce energy to the OM via TonB. Here, we structurally characterize the Ton complex from E. coli using X-ray crystallography, electron microscopy, DEER spectroscopy, and crosslinking, revealing a stoichiometry consisting of a pentamer of ExbB, a dimer of ExbD, and at least one TonB. Electrophysiology studies show that the Ton subcomplex forms $\mathrm{pH}$-sensitive cation-selective channels, providing insight to the mechanism by which it may harness the pmf for energy production. 